\title{
Histological activity and basal plasmacytosis are non- predictive markers for subsequent relapse in ulcerative colitis patients with mucosal healing
}

\author{
Farkas Klaudia1 ${ }^{1}$, Reisz Zita², Sejben Anita², Tiszlavicz László ${ }^{2}$, Szúcs Mónika ${ }^{3}$, Nyári Tibor ${ }^{3}$, \\ Szepes Zoltán ${ }^{1}$, Nagy Ferenc ${ }^{1}$, Rutka Mariann ${ }^{1}$, Bálint Anita1 $^{1}$, Bor Renáta ${ }^{1}$, Milassin Ágnes ${ }^{1}$, \\ Molnár Tamás ${ }^{1 *}$ \\ ${ }^{1} 1^{\text {st }}$ Department of Medicine, University of Szeged \\ ${ }^{2}$ Department of Pathology, University of Szeged \\ ${ }^{3}$ Department of Medical Physics and Informatics, University of Szeged
}

Received: February 05, 2016; Accepted: March 01, 2016; Published: March 30, 2016

*Corresponding author: Tamás Molnár, $1^{\text {st }}$ Department of Medicine, University of Szeged, H6720 Szeged, Koranyi fasor 8-10, Tel: +36-62545186; Fax: +36-62-545185; E-mail:molnar.tamas@med.u-szeged.hu

\begin{abstract}
Introduction: The assessment of histological remission started to become a more accurate and favorable therapeutic endpoint in ulcerative colitis (UC). This study aimed to investigate the rate of clinical relapse in UC patients after achieving mucosal healing and to evaluate the predictive role of basal plasmacytosis and microscopic inflammation on disease relapse.

Patients and methods: This was a prospective observational study conducted in UC patients with endoscopic remission defined by endoscopic Mayo (eMayo) subscore 0 or 1 . All patients underwent a colonoscopy and biopsy samples were taken the colonic mucosa. Follow-up appointments were months 6 , and 12. Microscopic inflammation was defined as a Geboes score $\geq 3.1$.

Results: Sixty-nine adult UC patients were enrolled in the study. Histology revealed basal plasmacytosis in $81.2 \%$ (focal plasmacytosis in $63.8 \%$, diffuse plasmacytosis in $17.4 \%$ of the cases) and microscopic inflammatory activity in $37.7 \%$ of patients with mucosal healing. At 6 and 12 months, clinical relapse occurred in $16.2 \%$, and in $14.5 \%$ of the patients. Geboes score $\geq 3.1$ and plasmacytosis detected in the biopsy samples at baseline were present in 9.1 and $72.7 \%$ of the patients samples relapsed at month 6, in 40 and $80 \%$ of the patients' samples relapsed at months 12 . Neither the presence of basal plasmacytosis, nor Geboes score $\geq 3.1$ was shown to be predictive of disease relapse.

Conclusion: The role of histological inflammation with mucosal healing requires more extensive research. Our results suggest that maintaining therapy after achieving endoscopic remission may influence the association between basal plasmacytosis and clinical relapse.
\end{abstract}

Keywords: Ulcerative colitis; Mucosal healing; Microscopic inflammation; Basal plasmacytosis; Relapse

\section{Introduction}

Mucosal healing has now been accepted as an essential target both in clinical trials and practice as well. Although many data support that mucosal healing is associated with prolonged clinical remission, lower rates of hospitalization and colectomy, recent studies came up with reverse data showing that mucosal healing does not predict sustained clinical remission in patients stopping biological therapy $[1,2]$. Since microscopic inflammation can even be detected in patients with mucosal healing, the assessment of histological remission started to become a more accurate and favorable therapeutic endpoint [3].

Geboes score including five features (architectural change, lamina propria neutrophils and eosinophils, neutrophils in epithelium, crypt destruction, and erosion or ulceration), is one of the best validated and most widely used index for the assessment of histological healing that was shown to predict relapse in ulcerative colitis (UC) patients achieving clinical and endoscopic remission [4, 5]. Moreover, Bitton et al. revealed that basal plasmacytosis, defined as the presence of plasma cells between the base of the crypts and the muscularis mucosae, was a significant predictor of relapse [6]. Since none of the histological scoring systems includes the assessment of basal plasmacytosis and limited data are available about the value of basal plasmacytosis and microscopic inflammation in quiescent UC, we aimed to investigate the rate of clinical relapse in UC patients after achieving mucosal healing and to evaluate the predictive role of basal plasmacytosis and microscopic inflammation on disease relapse.

\section{Patient and Method \\ Patient population}

This was a prospective observational study conducted in UC patients with endoscopic remission defined by endoscopic Mayo (eMayo) subscore 0 or 1 [7]. All patients underwent colonoscopy, and biopsy samples were taken from the uninflamed colonic mucosa. All colonic biopsies were separately evaluated by two 
expert pathologists for histologic activity (Geboes score) and the presence of basal plasmacytosis with focal or diffuse pattern. Basal plasmacytosis was defined as dense infiltrate of plasma cells around the deep part of the lamina propria or at the base of the crypts. C-reactive protein (CRP), partial Mayo (pMayo) scores and the used medications were documented at the time of the endoscopy, and the follow-up appointments: at months 6, and 12. Microscopic inflammation was defined as a Geboes score $\geq 3$.1 Disease relapse was defined as a partial Mayo (pMayo) score $\geq 3$. Alteration in CRP levels was compared to the baseline CRP values.

\section{Statistical analyses}

Multinominal logistic regression and linear mixed models were used to assess the predictive value of basal plasmacytosis and microscopic inflammation on disease relapse with evaluating the alteration of CRP and pMayo scores during the follow-up appointments. P-values $<0.05$ were considered significant for the analysis.

\section{Results}

Sixty-nine adult UC patients over 18 years of age with an endoscopically inactive disease and at least a 12-month follow-up were enrolled in the study. The median age at diagnosis was 31.4 years. The mean value of pMayo score at the time of the endoscopy was 0.65. Maintenance therapies at enrollment consisted of corticosteroids in $11.6 \%$, immunomodulators in $52.2 \%$ and biologicals in $18.8 \%$ of the patients. Demographic data are summarized in Table 1. Histology revealed basal plasmacytosis in $81.2 \%$ (focal plasmacytosis in $63.8 \%$, diffuse plasmacytosis in $17.4 \%$ of the cases) and microscopic inflammatory activity with a Geboes score $\geq 3.1$ in $37.7 \%$ of patients with mucosal healing. The mean time of follow-up was 1 year. At 6 and 12 months, clinical relapse occurred in $16.2 \%$, and in $14.5 \%$ of the patients.

Geboes score $\geq 3.1$ and plasmacytosis were detected in the biopsy samples at baseline histological examination in 9.1 and $72.7 \%$ of the patients who relapsed at month 6 and in 40 and $80 \%$ of the patients who relapsed at months 12 . In nonrelapsers, microscopic inflammation and basal plasmacytosis were detected at the baseline in 43.1 and $82.8 \%$ of the patients' samples at month 6, and in 44.2 and $82.7 \%$ at month 12 .

Neither the presence of basal plasmacytosis, nor Geboes score $\geq 3.1$ was shown to be predictive of disease relapse nor alteration in CRP levels at 6, and 12 months. No difference was observed if the data were analyzed separately in subgroups of eMayo score of 0 or 1 . Presence of basal plasmacytosis and microscopic inflammation in relapsers and nonrelapsers are shown in Figure 1 and Figure 2. Figure 3 contain the alterations of the medications during the examined period.

\section{Discussion}

Our results did not confirm the previous hypothesis that the presence of basal plasmacytosis and microscopic inflammation predicts UC clinical relapse in patients with mucosal healing at
Table 1: Demographic data of patients enrolled in the study.

\begin{tabular}{|c|c|}
\hline \multicolumn{2}{|c|}{ Table 1: Demographic data of patients enrolled in the study. } \\
\hline \multicolumn{2}{|c|}{ UC patients (n=69) } \\
\hline Mean age at diagnosis (years) & $31.4(11-60)$ \\
\hline Mean disease duration (years) & $17.8(1-58)$ \\
\hline Extent & $27 / 42$ \\
\hline Proctitis & \\
Left-sided colitis & 6 \\
Extensive colitis & 38 \\
\hline Disease activity at biopsy sample & 25 \\
\hline Inactive & \\
Mild & 45 \\
Moderate & 13 \\
Severe & 4 \\
\hline Mean CRP at biopsy sample (mg/dl) & 5 \\
\hline Concomitant medications & 4.2 \\
Corticosteroids & \\
Immunomodulators & 8 \\
Biologicals & 36 \\
Focal & 13 \\
\hline Diffuse & \\
Absent & 44 \\
Geboes score $\geq 3.1$ & 12 \\
\hline
\end{tabular}

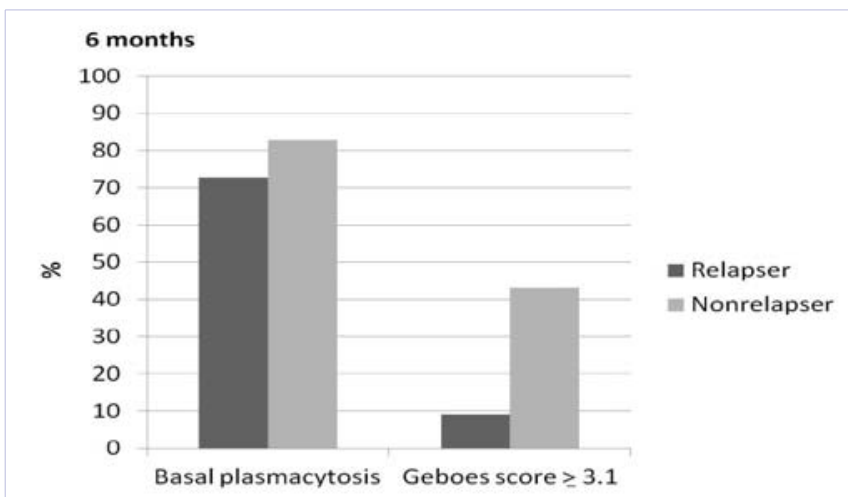

Figure 1: Presence of basal plasmacytosis and microscopic inflammation in relapsers and nonrelapsers at 6 month.

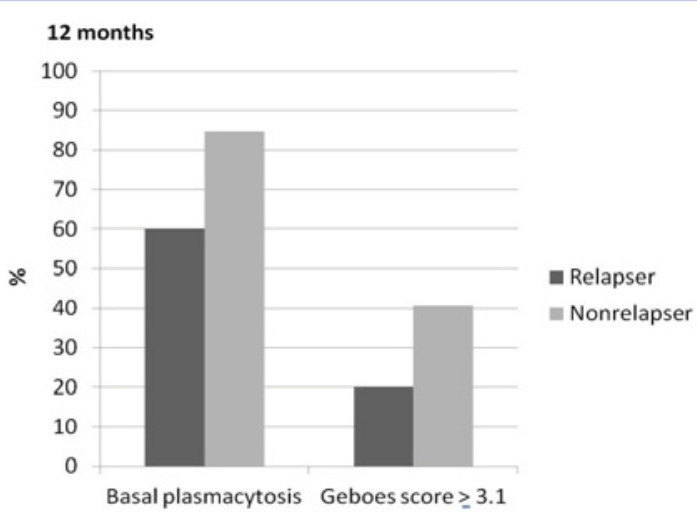

Figure 2: Presence of basal plasmacytosis and microscopic inflammation in relapsers and nonrelapsers at 12 month. 


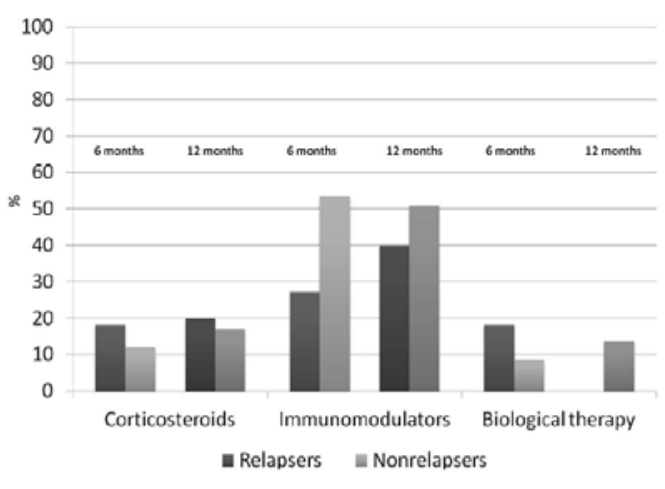

Figure 3: Alterations of the medications during the examined period.

any time points during 2 years. The overall rate of clinical relapse in our cohort was really low varying between 13 and $16 \%$ at the different examined periods. Focal and diffuse basal plasmacytosis were shown to be present in $63.8 \%$, and $17.4 \%$ of the patients in clinical and endoscopic remission. On the follow-up, basal plasmacytosis was detected in the biopsy samples at baseline histological examination in more than $70 \%$ of the relapsers at month 6 , and in $80 \%$ of them at month 12 . Therefore, specific histologic alteration did not predict nor short-term neither longterm relapse.

To date, only limited data are available on markers predictive to disease relapse in UC. Of the clinical parameters, age lower than 50-years old, previous relapses, extraintestinal manifestations, and low-fiber diet have been reported to be associated with higher relapse rates [8, 9]. Of biochemical markers, fecal calprotectin proved to be a strong predictive factor for UC flares [10].

Mucosal healing is a key goal of the clinical trials and the routine practice since it has been shown to be associated with better outcomes, improved quality of life and lower colectomy rates $[11,12]$. However, mucosal healing cannot completely exclude the presence of microscopic inflammation, which may play role in the development of complications and dysplasia occurrence [13]. Therefore, considering that histological healing is the only objective method for the assessment of mucosal healing in fact, achieving histological remission would be more favorable goal of therapy. Moreover, almost half of the UC patient with clinical and endoscopic remission has evidence of any histological inflammation [14]. Geboes score is an accepted score system for the evaluation of microscopic activity in the daily routine, however, basal plasmacytosis was also shown to be a promising marker for UC relapse.

Among the first studies, Bitton et al. revealed that beyond younger age and multiple previous relapses for women, basal plasmacytosis on rectal biopsy specimens was shown to be an independent predictor of earlier relapse [6]. The study by Villanacci et al. investigated the presence of basal plasmacytosis and its value in endoscopic biopsies from firstly diagnosed, non-treated "colitis" patients [15]. Their data proved that the presence of basal plasmacytosis in colonic endoscopic biopsies was a strong histological feature of IBD and helped in the differentiation from non IBD colitis. Basal plasmacytosis was always present in the ileum, cecum, and ascending colon, and variably present (17-60\%) in the other segments in $C D$, whereas in UC patients it was always present in the transverse and descending colon, and consistently present (77-87\%) in the other segments. The study by Bessissow et al. consisted of 75 UC patients confirmed that basal plasmacytosis, whether present in a focal or diffuse pattern, is an independent predictor of clinical relapse in UC patients with mucosal healing with a sensitivity, specificity, and accuracy of 47,85 , and $77 \%$, respectively. Inflammatory activity with a Geboes score $\geq 3.1$ was shown in $40 \%$ and basal plasmacytosis in $21 \%$ of the patients with endoscopic remission. At 12 months, clinical relapsed occurred in $20 \%$ of the patients. The study also revealed a strong trend toward a protective effect of biological therapy [5]. Comparing Bessissow's data with ours, the most remarkable difference is the higher rate of basal plasmacytosis in our cohort. Notably, focal plasmacytosis consisted almost two-third of our cases that may be explained by the extensive pathological assessment to detect plasma cells. The high proportion of focal plasmacytosis in our cohort also highlights the lack of correct definition of focal and diffuse plasmacytosis that may influence the statistical analysis in these works. In our study relapse rates also differed markedly, in the Bitton's study $36 \%$, in the Bessissow's study $20 \%$ of the patients relapsed. In our study the relapse rates were lower than in the above mentioned works despite of the high rate of basal plasmacytosis. Moreover, although maintenance therapy did not prove to be associated with the presence of basal plasmacytosis or microscopic inflammation in our relapsers, the type and the distribution of the medications differed in the two studies (etc. using steroids), therefore the role of concomitant therapy cannot be irrelevant when analyzing the results.

Although our results did not show that the presence of basal plasmacytosis and microscopic inflammation predicts UC clinical relapse in patients with mucosal healing, despite having a normal mucosa endoscopically, the role of histological inflammation with mucosal healing requires more extensive research. Our results suggest that maintaining therapy after achieving endoscopic remission may influence the association between basal plasmacytosis and clinical relapse.

\section{Take home messages}

Markers predictive to disease relapse in UC patients achieving mucosal healing are becoming more and more important. Our study did not confirm the predictive role of basal plasmacytosis and microscopic inflammation in clinical relapse in UC patients with endoscopic remission; however, maintenance therapy was suggested to influence the association between basal plasmacytosis and clinical relapse.

\section{Funding source}

This paper was supported by the János Bolyai Research Scholarship of the Hungarian Academy of Sciences (BO/00632/14/5). 


\section{References}

1. Dai C, Liu WX, Jiang M, Ming-Jun Sun. Mucosal healing did not predict sustained clinical remission in patients with IBD after discontinuation of one-year infliximab therapy. Plos One. 2014;9(10):e110797. doi:10.1371/journal.pone.0110797.

2. Farkas K, Lakatos PL, Szücs M, Pallagi-Kunstár É, Bálint A, Nagy F et al. Frequency and prognostic role of mucosal healing in patients with Crohn's disease and ulcerative colitis after one-year of biological therapy. World J Gastroenterol. 2014;20(11):2995-3001. doi:10.3748/wjg.v20.i11.2995.

3. Bryant RV, Winer S, Travis SPL, Riddell RH. Systematic review: Histological remission in inflammatory bowel disease. Is complete remission the new treatment paradigm? An IOIBD initiative. J Crohns Colitis. 2014;8(12):1582-1597. doi:10.1016/j.crohns.2014.08.011.

4. Geboes K, Riddell R, Ost A Jensfelt B, Persson T, Löfberg R. A reproducible grading scale for histological assessment of inflammation in ulcerative colitis. Gut. 2000;47(3):404-409.

5. Bessissow T, Lemmens B, Ferrante M, Bisschops R, Van Steen $\mathrm{K}$, Geboes K, et al. Prognostic value of serologic and histologic markers on clinical relapse in ulcerative colitis patients with mucosal healing. Am J Gastroenterol. 2012;107(11):1684-1692. doi:10.1038/ ajg.2012.301.

6. Bitton A, Peppercorn MA, Antonioli DA, Niles JL, Shah S, Bousvaros A, et al. Clinical, biological, and histologic parameters as predictors of relapse in ulcerative colitis. Gastroenterology. 2001;120(1):13-20.

7. Schroeder KW, Tremaine WJ, Ilstrup DM. Coated oral 5-aminosalicylic acid therapy for mildly to moderately active ulcerative colitis. A randomized study. N Engl J Med 1987;317(26):1625-1629.

8. Moum B, Ekbom A, Vatn MH, Aadland E, Sauar J, Lygren I, et al. Clinical course during the 1 st year after diagnosis in ulcerative colitis and Crohn's disease. Results of a large, prospective, population- based study in southeastern Norway, 1990-93. Scan J Gastroenterol. 1997;32(10):1005-1112.

9. Leo S, Leandro G, Di Matteo G, Caruso ML, Lorusso D. Ulcerative colitis in remission: is it possible to predict the risk of relapse? Digestion. 1989;44(4):217-221.

10. Costa F, Mumolo MG, Ceccarelli L, Bellini M, Romano MR, Sterpi $\mathrm{C}$, et al. Calprotectin is a stronger predictive marker of relapse in ulcerative colitis than in Crohn's disease. Gut. 2005;54(3):364-368. DOI:10.1136/gut.2004.043406.

11. Ardizzone S, Cassinotti A, Duca P, Mazzali C, Penati C, Manes $\mathrm{G}$, et al. Mucosal healing predicts late outcomes after the first course of corticosteroids for newly diagnosed ulcerative colitis. Clin Gastroenterol Hepatol. 2011;9(6):483-489. doi:10.1016/j. cgh.2010.12.028.

12. Solberg IC, Lygren I, Jahnsen J, Mazzali C, Penati C, Manes G, et al. Clinical course during the first 10 years of ulcerative colitis: results from a population-based inception cohort (IBSEN Study). Scand J Gastroenterol. 2009;44(4):431-440. doi:10.1080/00365520802600961.

13. Villanacci V, Antonelli E, Geboes K, Casella G, Bassotti G. Histological healing in inflammatory bowel disease: a still unfulfilled promise. World J Gastroenterol. 2013;19(7):968-978. doi:10.3748/wjg.v19. i7.968.

14. Tursi A, Elisei W, Picchio M, Forti G, Penna A, Inchingolo CD, et al. Histological inflammation in ulcerative colitis in deep remission under treatment with infliximab. Clinics and Research in Hepatology and Gastroenterology. 2015;39(1):107-113. doi:10.1016/j. clinre.2014.07.012.

15. Villanacci V, Antonelli E, Reboldi G, Salemme M, Casella G, Bassotti G.Endoscopic biopsy samples of naïve colitides patients: Role of basal plasmacytosis. J Crohns Colitis. 2014;8(11):1438-1443. doi:10.1016/j. crohns.2014.05.003. 